\title{
Un malato di rene policistico ha diritto all'invalidità e all'esenzione del ticket?
}

(da: www.renepolicistico.it)

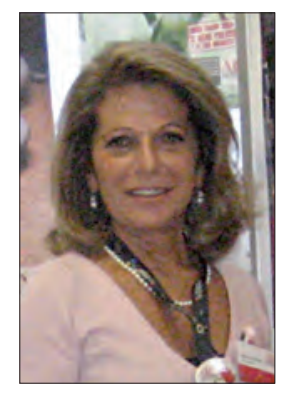

Luisa Sternfeld Pavia
La malattia renale policistica non gode delle esenzioni di cui al decreto istitutivo dei cosiddetti L.E.A. (livelli essenziali di assistenza), in quanto inspiegabilmente non è considerata una malattia cronica invalidante, se non quando evolve nell'insufficienza renale.

Esiste, però, un iter per poter ottenere un'esenzione parziale per medicinali e totale per analisi e indagini specialistiche e strumentali.

Premesso che la tabella che stabilisce il grado di invalidità destinato alle varie patologie attribuisce alla malattia del "Rene Policistico Bilaterale", al codice "6480", il $70 \%$ di invalidità fisso, è possibile richiedere l'invalidità parziale che, in base alla normativa regionale, se superiore al $67 \%$, dà diritto, appunto, all'esenzione succitata.

La procedura non è automatica e richiede qualche passaggio.

1. Recarsi dal proprio medico di base che dovrà redigere un certificato medico telematico apposito per richiesta di invalidità; sul certificato dovrà essere indicato chiaramente che il paziente soffre di malattia DEL RENE POLICISTICO AUTOSOMICO DOMINANTE (codice da indicare nel certificato che redige il medico: 75313) e deve descrivere chiaramente tutte le altre patologie eventuali e correlate.

2. Il medico invia telematicamente una copia del certificato all'INPS e una copia viene consegnata al paziente.

3. Il paziente con la copia del certificato deve recarsi presso un patronato (50 \& più, ACLI, ecc.), che gratuitamente redige la domanda di invalidità e la invia all'INPS.

4. Entro 30 o 60 giorni, il paziente viene convocato dalla commissione medica che deve valutare l'effettivo stato di salute; in quell'occasione è bene ripetere chiaramente

Accepted: May 4, 2016

Published online: August 3, 2016

Indirizzo per la corrispondenza:

Dr.ssa Luisa Sternfeld Pavia

AIRP Associazione Italiana Rene Policistico onlus

Via A. Bazzini 2

20131 Milano

luisa.sternfeld.airp@renepolicistico.it che si è affetti da una malattia genetica renale policistica autosomica dominante.

5. La commissione dovrebbe assegnare il 70\% (Tab. I) di invalidità anche senza riduzione della funzionalità renale, È possibile che la percentuale possa essere aumentata tenendo in considerazione eventuali altri problemi di salute.

6. Entro breve tempo, verrà comunicata la decisione della commissione; dopo si potrà ritirare, presso la ASL di competenza, il tesserino di esenzione da utilizzare presso il proprio medico di base per tutte le prescrizioni.

Si precisa che, contro la decisione della commissione, si potrà fare ricorso, entro i termini indicati nella lettera, qualora non risponda allo stato delle cose.

\section{Legge 104 e Legge 68, percentuali di invalidità e benefici (da: www.laleggepertutti.it)}

Quella che noi chiamiamo comunemente invalidità civile è una condizione prevista dal nostro ordinamento giuridico, correlata alla riduzione della capacità lavorativa di un individuo. Per compensare chi ha una ridotta capacità lavorativa, sono previste delle agevolazioni: in particolare, i benefici sono riconosciuti, in maniera differente, a partire dal $33.33 \%$ di invalidità. L'invalidità non deve essere confusa con l'handicap: il riconoscimento dell'handicap, secondo la Legge 104, è una condizione giuridica differente e aggiuntiva rispetto allo stato di invalido civile e dà diritto a benefici fiscali e ad agevolazioni lavorative diverse (come, per esempio, i permessi retribuiti). In questo breve vademecum, vediamo quali sono i benefici per l'invalidità, a seconda della riduzione della capacità lavorativa.

\section{Invalidità superiore al 33.33\%}

Bisogna innanzitutto precisare che lo status di invalido civile è riconosciuto solo a partire da una percentuale di riduzione della capacità lavorativa superiore a $1 / 3$, cioè al 33.33\%. Per la persona con invalidità superiore a tale soglia è previsto il diritto a protesi e ad ausili relativi alla patologia riconosciuta nel verbale di accertamento della commissione medica. La commissione medica, inoltre, può, indipendentemente dalla percentuale di invalidità, indicare sul verbale il diritto al Contrassegno per usufruire dei parcheggi per disabili. 
TABELLE I - Invalidità civile: scopri se hai diritto alla pensione di invalidità

\begin{tabular}{|c|c|c|c|c|}
\hline cod. & APPARATO URINARIO & $\min$. & $\max$. & fisso \\
\hline 6202 & CISTECTOMIA CON DERIVAZIONE NEL SIGMA & 41 & 50 & - \\
\hline 6204 & PROSTATITE CRONICA O IPERTROFIA PROSTATICA & 11 & 20 & - \\
\hline 6205 & RITENZIONE URINARIA CRONICA CON CATETERE A PERMANENZA & - & - & 46 \\
\hline 6206 & RITENZIONE URINARIA CRONICA CON CATETERISMO SALTUARIO & - & - & 25 \\
\hline 6208 & MEGAVESCICA & - & - & 30 \\
\hline 6401 & AGENESIA DI UN RENE NON COMPLICATA & - & - & 21 \\
\hline 6402 & ANOMALIE NON COMPLICATE DELLA PELVI RENALE & - & - & 21 \\
\hline 6422 & DUPLICITÀ OD ECTOPIA URETERALE BILATERALE & - & - & 41 \\
\hline 6423 & DUPLICITÀ OD ECTOPIA URETERALE MONOLATERALE & - & - & 15 \\
\hline 6451 & IPOPLASIA SEGMENTARIA RENALE SENZA DISTURBI FUNZIONALI & - & - & 15 \\
\hline 6462 & NEFRECTOMIA CON RENE SUPERSTITE INTEGRO & - & - & 25 \\
\hline 6463 & $\begin{array}{l}\text { NEFROLITIASI CON NECESSITÀ DI DIETA RIGIDA E DI ALMENO } 2 \text { CONTROLLI } \\
\text { E/O TRATTAMENTI ANNUALI }\end{array}$ & 21 & 30 & - \\
\hline 6473 & PTOSI RENALE BILATERALE NON COMPLICATA & - & - & 15 \\
\hline 6474 & RENE A FERRO DI CAVALLO CON CALCOLOSI & - & - & 45 \\
\hline 6475 & RENE A FERRO DI CAVALLO CON IDRONEFROSI & - & - & 55 \\
\hline 6476 & RENE A FERRO DI CAVALLO NON COMPLICATO & 11 & 20 & - \\
\hline 6477 & RENE ECTOPICO PELVICO & - & - & 11 \\
\hline 6478 & TUMORE DI WILMS & - & - & 95 \\
\hline 6479 & RENE MULTICISTICO UNILATERALE NON COMPLICATO & - & - & 21 \\
\hline 8207 & FISTOLA URETRALE & - & - & 15 \\
\hline 8208 & $\begin{array}{l}\text { CISTECTOMIA CON DERIVAZIONE ESTERNA O CON NEOVESCICA E SCARSO CONTROLLO } \\
\text { SFINTERICO }\end{array}$ & 51 & 60 & - \\
\hline 9203 & ESITI DI NEFROPATIA IN TRATTAMENTO DIALITICO PERMANENTE & 91 & 100 & - \\
\hline 9330 & TRAPIANTO RENALE & - & - & 60 \\
\hline
\end{tabular}

\section{Invalidità superiore al $45 \%$}

Chi possiede una percentuale di invalidità sopra il $45 \%$ ha la possibilità di usufruire del Collocamento Mirato. Per questi soggetti, nonché per i non vedenti e i sordomuti, è, infatti, previsto l'accesso ai servizi di sostegno e di collocamento dedicati: per usufruirne, gli interessati devono recarsi presso il centro per l'impiego, presentando, oltre al verbale di invalidità, la Relazione Conclusiva rilasciata dalla preposta commissione della ASL. Ricordiamo che possono iscriversi al Collocamento Mirato, senza dover richiedere la relazione conclusiva della ASL, anche gli invalidi del lavoro con una percentuale oltre il 33\%, gli invalidi di guerra, gli invalidi civili di guerra e gli invalidi per servizio con minorazioni ascritte dalla prima all'ottava categoria.

I lavoratori con invalidità civile superiore al $45 \%$ possono essere conteggiati dall'azienda nelle quote di riserva relative alla legge sul Collocamento obbligatorio, purché assunti almeno con un contratto part-time del $50 \%$ più un'ora (per esempio, considerando un orario ordinario di 40 ore settimanali, saranno sufficienti 21 ore a settimana). 


\section{Invalidità superiore al 51\%}

I lavoratori invalidi oltre il 51\% potranno usufruire di un congedo per cure relative all'infermità riconosciuta, per un periodo non superiore a 30 giorni all'anno. I costi sono, però, a carico dell'azienda, diversamente da quanto accade per i permessi della Legge 104, pertanto la possibilità di ottenere il permesso per invalidità va verificata all'interno del contratto collettivo di riferimento.

\section{Invalidità superiore al 60\%}

A partire da questa percentuale, il dipendente ha la possibilità di essere computato nella quota di riserva dell'impresa nella quale è già assunto, a prescindere dall'orario del contratto. Il beneficio non è riconosciuto se l'inabilità è stata causata da un inadempimento del datore di lavoro.

\section{Invalidità superiore al $74 \%$}

Gli invalidi civili sopra il $74 \%$ hanno diritto a un assegno di assistenza, concesso dai 18 ai 65 anni, il cui importo è di 279 euro mensili per il 2015, con un limite di reddito di 4805.19 euro.

L'assegno di invalidità civile non richiede, come l'assegno di invalidità ordinaria (categoria $I O$ ) il pagamento di un minimo di contributi all'INPS; la prestazione è incompatibile con qualsiasi pensione diretta di invalidità a carico dell'Ago (assicurazione generale obbligatoria) e con tutte le prestazioni pensionistiche di invalidità per causa di guerra, di lavoro o di servizio, comprese le rendite Inail. L'interessato può, comunque, optare per il trattamento più favorevole.

\section{Invalidità superiore al $75 \%$}

Per chi ha un'invalidità sopra il $\mathbf{7 5 \%}$ sono previsti dei benefici pensionistici: nel dettaglio, per ogni anno lavorato, sono accreditati 2 mesi di contributi in più, sino a un massimo di 5 anni. L'agevolazione può essere riconosciuta dal 2002 in poi.

\section{Invalidità superiore all'80\%}

Per coloro che hanno un'invalidità dell' $80 \%$ e oltre, è previsto, grazie alla Deroga Amato, l'accesso anticipato alla pensione di vecchiaia, con 55 anni e 3 mesi d'età per le donne e 60 anni e 3 mesi per gli uomini (dal 2016 i requisiti saranno 55 anni e 7 mesi e 60 anni e 7 mesi).

\section{$100 \%$ di invalidità}

Chi è invalido al $\mathbf{1 0 0 \%}$ può fruire dei seguenti benefici:

- $\quad$ esenzione dal ticket per le prestazioni mediche specialistiche e diagnostiche e per i medicinali;

- $\quad$ pensione di inabilità, concessa a chi ha un reddito sino a 16532.10 euro e compatibile, sino al limite di reddito, con l'assegno ordinario di invalidità o la pensione di invalidità mensile.

\section{Assegno di accompagnamento}

Si ha diritto all'indennità di accompagnamento, pari a circa 500 euro mensili (l'importo viene adeguato annualmente). L'assegno è riconosciuto, indipendentemente al reddito, a chi ha difficoltà a compiere gli atti quotidiani della vita o a chi non può deambulare senza l'aiuto di un'altra persona.

\section{Disclosures}

Financial support: No financial support was received for this submission.

Conflict of interest: The author has no conflict of interest. 